OPEN ACCESS

Edited by:

Hubert Vaudry,

University of Rouen, France

Reviewed by:

Etienne Challet,

Centre National de la Recherche

Scientifique, France

Fabrice Morin,

University of Rouen, France

*Correspondence:

Saumen Kumar Maitra,

Department of Zoology,

Visva-Bharati University,

Santiniketan - 731235, India

dgp_skmaitra@yahoo.co.in

Specialty section:

This article was submitted to Neuroendocrine Science, a section of

the journal

Frontiers in Endocrinology

Received: 19 December 2014 Accepted: 07 July 2015

Published: 22 July 2015

Citation:

Mukherjee S and Maitra SK (2015) Gut melatonin in vertebrates: chronobiology and physiology.

Front. Endocrinol. 6:112. doi: 10.3389/fendo.2015.00112

\section{Gut melatonin in vertebrates: chronobiology and physiology}

\author{
Sourav Mukherjee and Saumen Kumar Maitra* \\ Department of Zoology, Visva-Bharati University, Santiniketan, India
}

Melatonin, following discovery in the bovine pineal gland, has been detected in several extra-pineal sources including gastrointestinal tract or gut. Arylalkylamine $N$ acetyltransferase (AANAT) is the key regulator of its biosynthesis. Melatonin in pineal is rhythmically produced with a nocturnal peak in synchronization with environmental light-dark cycle. A recent study on carp reported first that melatonin levels and intensity of a $23 \mathrm{kDa}$ AANAT protein in each gut segment also exhibit significant daily variations but, unlike pineal, show a peak at midday in all seasons. Extensive experimental studies ruled out direct role of light-dark conditions in determining temporal pattern of gut melatoninergic system in carp, and opened up possible role of environmental non-photic cue(s) as its synchronizer. Based on mammalian findings, physiological significance of gut-derived melatonin also appears unique because its actions at local levels sharing paracrine and/or autocrine functions have been emphasized. The purpose of this mini review is to summarize the existing data on the chronobiology and physiology of gut melatonin and to emphasize their relation with the same hormone derived in the pineal in vertebrates including fish.

Keywords: gut melatonin, AANAT, chronobiology, physiology

\section{Introduction}

Extensive research carried out in past 50 years have clearly depicted melatonin (5-methoxy- $\mathrm{N}$ acetyltryptamine) as a potent chronobiotic molecule involved in the regulation of a variety of physiological functions (1). Following discovery in the bovine pineal gland (2), melatonin is detected in several non-pineal tissues/organs, such as retina, Harderian gland, and gastrointestinal tract (GIT) or gut. However, existing knowledge on melatonin has stemmed largely from the studies on pineal that too in mammals, leaving non-pineal tissues, especially in lower vertebrates, as an interesting topic of research. Though functional characterization of melatonin in gut, relative to that in retina and Harderian gland, has received serious attention, the studies are limited mostly to the mammals, especially rodents and pigs. Moreover, current knowledge on the cellular localization, distribution, and temporal pattern of gut melatonin is scarce and inconsistent as well. Thus, a brief review of the existing literature on gut melatonin appears meaningful for understanding its unique features in vertebrates.

\section{Cellular Localization and Distribution}

Localization of melatonin in the enterochromaffin cells (EC) of digestive mucosa of rat was followed by its quantitative estimation in gut tissues. At the sub-cellular level, strongest binding was noted in nuclear fraction, followed by microsomal, mitochondrial, and cytosolic fractions (3). 
In mammals, melatonin-producing cells were found in submucosa and muscularis layer of esophagus, the glandular portion of the gastric wall and in the area of Lieberkühn's crypts and Brunner's glands of duodenum, and more specifically in EC of mucosal layer (3). As in birds and mammals, maximum immunoreactivity was noted at the outer margin of lamina propria in mucosal layer of intestinal villi in carp gut (4).

Generally, melatonin concentrations in gut tissues surpass the levels of melatonin in circulation by 10-100 times (3). Daytime levels of gut melatonin were measured in several species (5) of fish, e.g., sturgeon, rainbow trout, and carp (stomach $\sim 102 \mathrm{pg} / \mathrm{g}$, proximal gut or PG $\sim 146 \mathrm{pg} / \mathrm{g}$, and distal gut or DG $\sim 141 \mathrm{pg} / \mathrm{g}$ ); amphibians, e.g., axolotl (stomach and PG $\sim 44 \mathrm{pg} / \mathrm{g}$ and DG $\sim 92 \mathrm{pg} / \mathrm{g}$ ), and bullfrog (esophagus $\sim 73 \mathrm{pg} / \mathrm{g}$, stomach $\sim 78 \mathrm{pg} / \mathrm{g}$, PG $\sim 20 \mathrm{pg} / \mathrm{g}$, and DG $\sim 152 \mathrm{pg} / \mathrm{g}$ ); and reptiles, e.g., red-sided garter snake (stomach $\sim 1018 \mathrm{pg} / \mathrm{g}, \mathrm{PG} \sim 328 \mathrm{pg} / \mathrm{g}$, and DG $\sim 511 \mathrm{pg} / \mathrm{g})$. The midday values of melatonin in the anterior $(\sim 550 \mathrm{pg} / \mathrm{g})$, middle $(\sim 538 \mathrm{pg} / \mathrm{g})$, and posterior $(\sim 578 \mathrm{pg} / \mathrm{g})$ segments of gut in a day-active carp (6) did not indicate any regional variations in its distribution in the same animal, but depicted species-specific variations in a particular gut segment (5). However, it remains obscure whether gut melatonin levels vary between the day-active and night-active animals.

\section{Biosynthesis of Melatonin}

The synthesis of melatonin in all the melatonin-synthesizing cells is a four-step phenomenon. First, the precursor L-tryptophan is taken up from the circulation (blood) and is converted to 5-hydroxy-tryptophan (5-HTP) in the mitochondria by Trp5 -mono-oxygenase/hydroxylase and is then decarboxylated in the cytosol by L-aromatic amino acid decarboxylase to form serotonin (5-hydroxytryptamine, 5-HT), that in turn is acetylated $(N$-acetylation) into $N$-acetyl serotonin by arylalkylamine$N$-acetyltransferase (AANAT) (7), which is considered as the rate-limiting enzyme in melatonin biosynthetic pathway. Finally, $\mathrm{N}$-acetyl serotonin is $\mathrm{O}$-methylated by hydroxyindole-Omethyltransferase (HIOMT) to form melatonin (8).

Endogenous melatonin biosynthesis within the ECs of the digestive mucosa has been evident from the studies on the expression of genes for two key melatonin-synthesizing enzymes. The study of gut tissues detected mRNA expression of Aanat in rat (9) and Hiomt in quail (10) as well as goldfish (11). Aanat-2 expression, as noted in the gut of goldfish (11) and rainbow trout (12), was supported by densitometric analysis of AANAT protein in the carp gut (6). The study detected a $\sim 23 \mathrm{kDa}$ AANAT protein corresponding to AANAT in the pineal of pike, trout, and carp (13) in gut tissue homogenates and thereby ensured endogenous synthesis of melatonin in gut.

\section{Chronobiology}

\section{Temporal Pattern of Gut Melatonin}

Circulating profiles of melatonin in different vertebrates (14), including carp (15), exhibit precise diurnal rhythms with a peak during the dark phase and nadir during the photo phase and such rhythms are primarily generated by pineal AANAT (7). Until recently, comparable data on the temporal pattern of gut melatonin and its regulatory mechanisms were unknown for any vertebrates. A study on mice reported lower melatonin level during the day relative to nocturnal values only in the duodenum-jejunum segment of the GIT (16). In goldfish, though titers of melatonin were not measured, analysis of Aanat-2 mRNA expression revealed a daily rhythm in hindgut, but not in foregut (11). Importantly, the daily peak in the Aanat-2 mRNA expression persisted under continuous light as well as continuous darkness (11). Conversely, the study on carp by showing parallel changes in the levels of melatonin and AANAT density for the first time demonstrated that melatonin-synthesizing system in each gut segment, irrespective of seasons, undergoes significant daily variations with a peak at midday (6). Such findings also opened up a possibility that regulatory mechanism of melatonin synthesis in gut and pineal in the same animal species (Figure 1) is different (13).

\section{Influence of Photoperiods on Rhythmicity in Gut Melatonin}

The duration of light and thereby duration of darkness in a 24$\mathrm{h}$ cycle is known to play a key role in the regulation of melatonin synthesis in the pineal (14). It is noteworthy that in fish, including carp (13), light acts directly on the pineal gland. By contrast, in mammals, light is detected by the retina and affects the pineal gland indirectly with a multisynaptic pathway (14). However, the question whether lighting conditions play a critical role of a synchronizer or regulator of gut melatonin was not duly addressed. Thus, a study was undertaken with carp (17), which due to its natural surface dwelling habit maintains close contact with environmental light and thereby is considered as an ideal model for the study of photo-response mechanisms in any melatonin-synthesizing tissue. Accordingly, the carp were held either under altered long (LP) or short photoperiods (SP) in a 24-h cycle, or under continuous light (LL) or darkness (DD) for equal duration. The results of the study indicated that none of the employed photo-regimens has any significant effect per se on the daily profiles of gut melatonin levels and AANAT protein density (17). Notably, an earlier study on the same carp demonstrated that diurnal rhythmicity in serum melatonin and pineal AANAT with a nocturnal peak (13) was lost when fish were held under LL or DD. Taken together, it is suggested that melatonin synthesis in gut, unlike pineal, is not a dark-dependent phenomenon, and environmental lighting conditions may not serve the role of external cue(s) in determining its rhythmic features in a daily cycle.

\section{Influences of Food on Rhythmicity in Gut Melatonin}

One of the periodic variables in the gut is the availability of food, which may serve as an important cue to determine daily periodicity of melatonin synthesis in gut (18). Notably, the study on the distribution of melatonin in different parts of the GIT in cow (poly-gastric) and pig (mono-gastric) revealed that cows had higher melatonin levels in stomach and ileum, but lower in cecum and colon (19). Accordingly, a relation between melatonin secretion and the digestive functions is sought. Depending on the meal frequency and timing of meals, several circulating metabolites and hormones undergo daily variations (20), and these variations 


\section{Temporal pattern of gut and serum melatonin profiles in carp (Catla catla) and light-dark conditions of the environment}

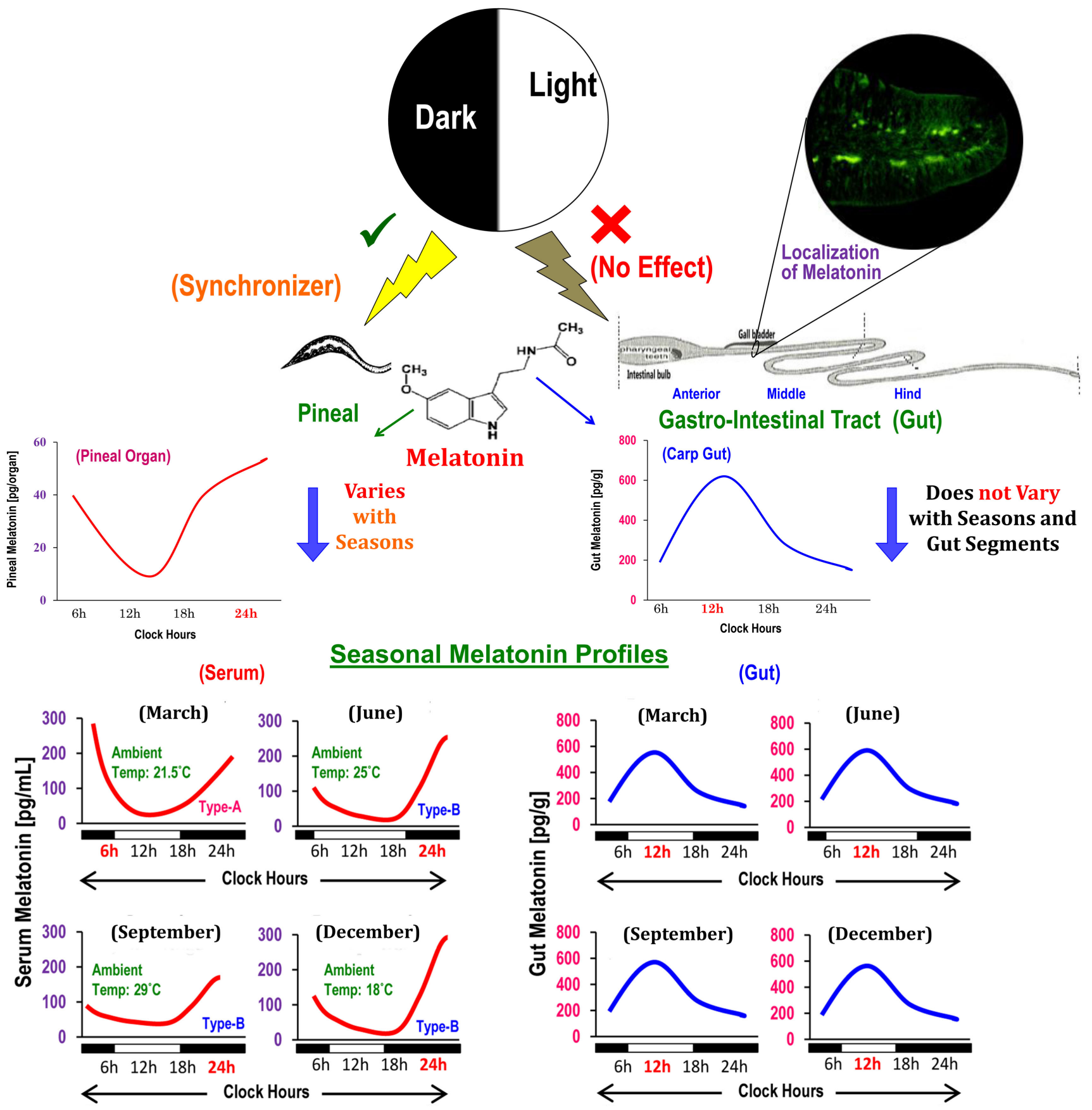

FIGURE 1 | Schematic presentation of the summary of existing information on the localization, distribution, and temporal organization of melatonin concentrations in different anatomical segments of gut in relation to the diurnal and seasonal profiles of serum melatonin in carp Catla catla and light-dark cycle in the environment.

are dependent on whether the animal is fed, fasted, or starved. Because timing of food intake is roughly opposite in phase between diurnal and nocturnal species, it is likely that synthesis of gut melatonin is correlated with feeding in various animals including fish species. However, any data from appropriate experimental studies that could support the hypothesis on a direct role of food availability and its timings on gut melatoninergic system in any animal species still remain unknown. 


\section{Physiology of Gut Melatonin}

Melatonin is a lipophilic compound diffusing rapidly through biological membranes and actions in an endocrine and/or, paracrine and/or, autocrine manners. It is involved in the regulation of multiple functions, including the control of gastrointestinal system. Melatonin produced by EC may perform paracrine functions, while its action in the intestinal muscles may be either direct or it may act via the myenteric nervous system $(3,19)$.

A study on rainbow trout demonstrated that melatonin is released from gastrointestinal tissue and addition of L-tryptophan to the incubation medium stimulates melatonin synthesis and release (21). The presence of melatonin in intestinal villi indicated that melatonin might be involved in the transmembrane transport of electrolytes and ions. Moreover, melatonin increased water content in feces (22) and the topical application of melatonin stimulated short-circuit current in colonic cells (23). Experimental studies confirmed that melatonin also inhibited the contraction of smooth muscles in stomach, ileum, and colon (24). In rats, endogenous melatonin affected the electro-myogram of pre- and post-prandial motility, though such actions were observed only at night (25), when the concentration of melatonin in blood remained usually high. Perhaps, melatonin relaxes gastrointestinal muscles by specifically blocking nicotinic channels (26).

The assumption that melatonin may also interact with dopamine-sensitive, possibly $\mathrm{Ca}^{2+}$-activated, $\mathrm{K}^{+}$channels (27) earned support from the study, in which inhibition of smallconductance $\mathrm{K}^{+}$channels attenuated melatonin-induced relaxation of gastric muscles (28). In addition to its relaxation effect presented in several in vitro studies, melatonin appeared to inhibit serotonin (5-HT) action also in vivo. Serotonin facilitated the "food transit time" (FTT) (the moment of food intake to the appearance of the first feces stained with food coloring), as compared to controls, whereas injection of melatonin to serotonin implanted mice significantly increased the FTT (19). In a similar study, small doses of melatonin relaxed the gut muscles and facilitated intestinal motility in rats (19). Further studies argued that a counterbalancing system exists between serotonin and melatonin which within the GIT melatonin functions as a physiological antagonist of serotonin (22). High dose of melatonin inhibited the spontaneous or serotonin-induced contraction of GIT muscles and induced intestinal elongation. Conversely, low doses of melatonin stimulated intestinal contraction, resulting in the shortening of gut (3).

Several mammalian studies indicated that melatonin may have a protective role against development of gastric ulcers $(29,30)$. It is proposed that the prevention of stress- or ethanol-induced gastric lesions in rats is probably due to the anti-serotoninergic effect of melatonin (29). The incidence and severity of spontaneously induced gastric ulcers are significantly reduced in pigs, which were

\section{References}

1. Chowdhury I, Maitra SK. Chapter 1: melatonin in the promotion of health. In: Watson RR, editor. Melatonin Time Line: From Discovery to Therapy. Boca Raton, FL: Taylor and Francis (2012). p. 1-60. fed with $5 \mathrm{mg}$ melatonin/kg enriched food. The highest incidence of ulcers is observed in pigs with the lowest level of melatonin in their plasma and stomach tissues (30). Protection against stressinduced lesions might be due to a strong antioxidant action of melatonin and the restoration of microcirculation (31).

In addition to its antioxidant effect, melatonin action in the prevention or treatment of colitis (32) includes the stimulation of the immune system. Though direct evidence is lacking, indications are available to show that melatonin administration to rats significantly increased the number and size of Peyer's patches, the major immune tissue of the GIT (33). Melatonin treatment may improve irritable bowel symptoms (IBS) as well (34). Thus, over the decades, melatonin has been promoted as a "magic cure" for the treatment or prevention of several physiological disorders ranging from aging to aggression, depression to hypertension, suppressed immunity to oxidative stress, insomnia to jet lag (1). However, convincing data are yet to be known to prove that such actions are also ascribed to gut-derived melatonin.

\section{Conclusion}

The information gathered so far provides indications that environmental and neuroendocrine regulatory mechanisms of melatonin synthesis in the pineal and in the gut are different. Likewise, functional characteristics of pineal-derived and GIT-derived melatonin may not be identical, as the nature of release and function of this extra-pineal melatonin are not yet fully understood. There are reasons to argue that pineal melatonin mostly acts as an endocrine agent, whereas GIT melatonin performs not only endocrine functions but also in autocrine and paracrine manners (18). However, emergence of a general idea on the physiological significance of gut melatonin has suffered a major setback due to lack of data from studies on any non-mammalian species and thereby warrants further carefully controlled research under diverse experimental conditions using animals representing different groups of vertebrates.

\section{Acknowledgments}

Financial assistance from the Council of Scientific and Industrial Research [37(1390)/09/EMR-II] and the Department of Biotechnology (BT/PR11423/AAQ/03/421/2008), Govt. of India, New Delhi to SKM, and a Research Associateship (A/F/4S/2786/2015) by the Department of Biotechnology, and an international travel grant from the Department of Science and Technology (SB/ITS/02594/2014-15), Govt. of India to SM, are thankfully acknowledged. The authors are grateful to the editor and two reviewers for their critique and invaluable comments on the previous version of the manuscript.

2. Lerner AB, Case JD, Takahashi Y, Lee TH, Mori N. Isolation of melatonin, pineal factor that lightens melanocytes. J Am Chem Soc (1958) 80:2587. doi:10. 1021/ja01543a060

3. Bubenik GA. Gastrointestinal melatonin localization, function, and clinical relevance. Dig Dis Sci (2002) 47:2336-48. doi:10.1023/A:1020107915919 
4. Mukherjee S. Gut Melatonin in Carp Catla catla: Distribution, Daily Profiles in Relation to Photoperiods, Food and Feeding Schedule. Ph.D. thesis, Visva-Bharati, Santiniketan, India (2014).

5. Bubenik GA, Pang SF. Melatonin level in the gastrointestinal tissue of fish, amphibians, and a reptile. Gen Comp Endocrinol (1997) 106:415-9. doi:10.1006/ gcen.1997.6889

6. Mukherjee S, Moniruzzaman M, Maitra SK. Daily and seasonal profiles of gut melatonin and their temporal relationship with pineal and serum melatonin in carp Catla catla under natural photo-thermal conditions. Biol Rhythm Res (2014) 45:301-15. doi:10.1080/09291016.2013.817139

7. Voisin P, Namboodiri MAA, Klein DC. Arylamine N-acetyltransferase and arylalkylamine $N$-acetyltransferase in the mammalian pineal gland. J Biol Chem (1984) 259:10913-8.

8. Axelrod J, Weissbach H. Enzymatic O-methylation of $\mathrm{N}$-acetylserotonin to melatonin. Science (1960) 131:1312. doi:10.1126/science.131.3409.1312

9. Stefulj J, Hörtner M, Ghosh M, Schauenstein K, Rinner I, Wölfler A, et al. Gene expression of the key enzymes of melatonin synthesis in extrapineal tissues of the rat. J Pineal Res (2001) 30:243-7. doi:10.1034/j.1600-079X.2001.300408.x

10. Hong GX, Pang SF. N-Acetyltransferase activity in the quail (Cotornix coturnix jap) duodenum. Comp Biochem Physiol (1995) 112:251-5. doi:10.1016/ 0305-0491(95)00083-6

11. Velarde E, Cerdá-Reverter JM, Alonso-Gómez AL, Sánchez E, Isorna E, Delgado MJ. Melatonin-synthesizing enzymes in pineal, retina, liver, and gut of the goldfish (Carassius): mRNA expression pattern and regulation of daily rhythms by lighting conditions. Chronobiol Int (2010) 27:1178-201. doi:10. 3109/07420528.2010.496911

12. Fernández-Durán B, Ruibal C, Polakof S, Ceinos RM, Soengas JL, Míguez JM. Evidence for arylalkylamine N-acetyltransferase (AANAT2) expression in rainbow trout peripheral tissues with emphasis in the gastrointestinal tract. Gen Comp Endocrinol (2007) 152:289-94. doi:10.1016/j.ygcen.2006.12.008

13. Seth M, Maitra SK. Importance of light in temporal organization of photoreceptor proteins and melatonin-producing system in the pineal of carp Catla catla. Chronobiol Int (2010) 27:463-86. doi:10.3109/07420521003666416

14. Falcón J, Migaud H, Muñoz-Cueto JA, Carrillo M. Current knowledge on the melatonin system in teleost fish. Gen Comp Endocrinol (2010) 165:469-82. doi:10.1016/j.ygcen.2009.04.026

15. Maitra SK, Chattoraj A, Bhattacharyya S. Implication of melatonin in oocyte maturation in Indian major carp Catla catla. Fish Physiol Biochem (2005) 31:201-7. doi:10.1007/s10695-006-0025-2

16. Bubenik GA, Niles LP, Pang SF, Pentney PJ. Diurnal variation and binding characteristics of melatonin in the mouse brain and gastrointestinal tissues. Comp Biochem Physiol (1993) 104:221-4.

17. Mukherjee S, Moniruzzaman M, Maitra SK. Impact of artificial lighting conditions on the diurnal profiles of gut melatonin in a surface dwelling carp (Catla catla). Biol Rhythm Res (2014) 45:831-48. doi:10.1080/09291016.2014.923618

18. Vera LM, De Pedro N, Gómez-Milán E, Delgado MJ, Sánchez-Muros MJ, Madrid JA, et al. Feeding entrainment of locomotor activity rhythms, digestive enzymes and neuroendocrine factors in goldfish. Physiol Behav (2007) 90:518-24. doi:10.1016/j.physbeh.2006.10.017

19. Bubenik GA. Thirty four years since the discovery of gastrointestinal melatonin. J Physiol Pharmacol (2008) 59:33-51.

20. Boujard T, Leatherland JF. Circadian rhythms and feeding time in fishes. Environ Biol Fish (1992) 35:109-31. doi:10.1007/BF00002186

21. Lepage O, Larson ET, Mayer I, Winberg S. Tryptophan affects both gastrointestinal melatonin production and interrenal activity in stressed and nonstressed rainbow trout. J Pineal Res (2005) 38:264-71. doi:10.1111/j. 1600-079X.2004.00201.x

22. Bubenik GA, Pang SF. The role of serotonin and melatonin in the gastrointestinal physiology: ontogeny, regulation of food intake and mutual 5-HT, melatonin feedbacks. J Pineal Res (1994) 16:91-9. doi:10.1111/j.1600-079X. 1994.tb00088.x

23. Chan H, Lui K, Wong W, Poon A. Effect of melatonin on chloride secretion by human colonic $\mathrm{T}_{84}$ cells. Life Sci (1998) 23:2151-8. doi:10.1016/S0024-3205(98) 00190-8

24. Harlow HJ, Weekly BL. Effect of melatonin on the force of spontaneous contractions of in vitro rat small and large intestines. J Pineal Res (1986) 3:277-84. doi:10.1111/j.1600-079X.1986.tb00750.x

25. Merle A, Delagrange PH, Renard P, Lesieur D, Cuber JC, Roche M, et al. Effect of melatonin on motility pattern of small intestine in rats and its inhibition by melatonin receptor antagonist S 221152. J Pineal Res (2000) 29:116-24. doi:10.1034/j.1600-079X.2000.290208.x

26. Barajas-Lopez C, Peres AL, Espinosa-Luna R, Reyes-Vazquez C, Prieto-Gomez B. Melatonin modulates cholinergic transmission by blocking nicotinic channels in the guineapig submucous plexus. Eur J Pharmacol (1996) 312:319-25 doi:10.1016/0014-2999(96)00481-5

27. Reyes-Vasquez C, Naranjo-Rodriguez EB, Garcia-Segoviano JA, TrujilloSantana J, Prieto-Gomez B. Apamin blocks the direct relaxant effect of melatonin on rat ileal smooth muscles. J Pineal Res (1997) 22:1-8. doi:10.1111/j. 1600-079X.1997.tb00295.x

28. Storr M, Schudziarra V, Allescher H-D. Inhibition of small conductance $\mathrm{K}^{+}$channels attenuated melatonin-induced relaxation of serotonin-contracted rat gastric fundus. Can J Physiol Pharmacol (2000) 78:799-806. doi:10.1139/ y00-059

29. Cho CH, Pang SF, Chen BW, Pfeiffer CJ. Modulating action of melatonin on serotonin-induced aggravation of ethanol ulceration and changes of mucosal blood flow in rat stomach. J Pineal Res (1989) 6:89-97. doi:10.1111/j. 1600-079X.1989.tb00406.x

30. Bubenik GA, Ayles HL, Ball RO, Friendship RM, Brown GM. Relationship between melatonin levels in plasma and gastrointestinal tissues and the incidence and severity of gastric ulcers in pigs. J Pineal Res (1998) 24:62-6. doi:10. 1111/j.1600-079X.1998.tb00367.x

31. Konturek PC, Brzozowski T, Konturek SJ. Gut clock: implication of circadian rhythms in the gastrointestinal tract. J Physiol Pharmacol (2011) 62:139-50.

32. Pentney P, Bubenik GA. Melatonin reduces the severity of dextran-induced colitis in mice. J Pineal Res (1995) 19:31-9. doi:10.1111/j.1600-079X.1995. tb00168.x

33. Yanagisawa M, Kachi T. Effects of the pineal hormone on Payer's patches in the small intestine. Acta Anat Nippon (1994) 69:522-7.

34. Elsenbruch S. Melatonin: a novel treatment for IBS? Gut (2005) 10:1353-4. doi:10.1136/gut.2005.074377

Conflict of Interest Statement: The authors declare that the research was conducted in the absence of any commercial or financial relationships that could be construed as a potential conflict of interest.

Copyright () 2015 Mukherjee and Maitra. This is an open-access article distributed under the terms of the Creative Commons Attribution License (CC BY). The use, distribution or reproduction in other forums is permitted, provided the original author(s) or licensor are credited and that the original publication in this journal is cited, in accordance with accepted academic practice. No use, distribution or reproduction is permitted which does not comply with these terms. 